\title{
Green and Sustainable Heterogeneous Organo-Catalyst for Asymmetric Aldol Reactions
}

\author{
Mohammad Sadiq1 ${ }^{*}$, Razia Aman', Khalid Saeed1, M. Sohail Ahmad1', M. Abid Zia ${ }^{2}$ \\ ${ }^{1}$ Department of Chemistry, University of Malakand, Malakand, Pakistan \\ ${ }^{2}$ Department of Chemistry, University of Education Attock Campus, Attock, Pakistan \\ Email: ${ }^{*}$ mohammad_sadiq26@yahoo.com, , sadiq@uom.edu.pk
}

Received 27 January 2015; accepted 22 March 2015; published 30 March 2015

Copyright @ 2015 by authors and Scientific Research Publishing Inc.

This work is licensed under the Creative Commons Attribution International License (CC BY). http://creativecommons.org/licenses/by/4.0/

(c) (i) Open Access

\begin{abstract}
Linear amino acids covalently supported on graphene sheet were employed as catalysts for asymmetric aldol reactions between cyclohexanone and aldehydes (aliphatic and/or aromatic) in a batch type reactor in the presence of water. The reactions were found to exhibit high yield as well as excellent ee value. Additionally, the catalysts were found to be truly heterogeneous and eco-friendly.
\end{abstract}

Keywords

Immobilization, Graphene Sheet, Enantioselectivity, Cyclohexanone, Aldehyde

\section{Introduction}

Recently, linear amino acids have been extensively investigated as effective homogenous catalysts in asymmetric aldol reactions between ketone and aldehyde in the presence of polar solvents [1]-[3]. Several problems are usually encountered in these homogeneous catalytic systems, which include the presence of a suitable polar solvent, large catalyst loading and the catalyst's regeneration and its extended life span [4]. To answer the issues inherent to the use of amino acids in homogeneous systems, researchers intensively exploited suitable alternatives to replace these environmentally malignant systems [5]. In this scenario, immobilization of amino acids on a suitable support could simplify the separation of products, and facilitate the recovery and reusability of the catalysts [6]. A great deal of research has been focused on the use of immobilized amino acids as heterogeneous catalysts, such as poly (vinyl alcohol)-graft-poly (ethylene glycol)-supported hydroxyl proline for aldol reactions

*Corresponding author.

How to cite this paper: Sadiq, M., Aman, R., Saeed, K., Ahmad, M.S. and Zia, M.A. (2015) Green and Sustainable Heterogeneous Organo-Catalyst for Asymmetric Aldol Reactions. Modern Research in Catalysis, 4, 43-49.

http://dx.doi.org/10.4236/mrc.2015.42006 
with stereo selectively in a variety of solvents, as well as in neat substrates [7]. Similarly, Henseler et al. [8] has demonstrated the use of polystyrene-supported threonine for asymmetric aldol reactions, with satisfactory yield and ee value. The recovery of the catalyst without loss of its activity and its subsequent re-usability is an important finding in both studies. These studies motivated us to investigate the efficiency and capability of covalently immobilized linear amino acids on graphene sheet for asymmetric aldol reactions between cyclohexanone and aromatic and aliphatic aldehydes under eco-friendly reaction conditions.

\section{Experimental}

\subsection{Preparation of Catalysts}

Graphene sheet (supplied by Chengdu Organic Chemicals Co. Ltd.) were functionalized by modified Hummer and Offeman's method [9]. $1 \mathrm{~g}$ of graphene sheet was sonicated in $50 \mathrm{~mL}$ solution of $\mathrm{HNO}_{3}(5 \mathrm{M})$ and $\mathrm{H}_{2} \mathrm{SO}_{4}(5$ $\mathrm{M})$ in the presence of $\mathrm{KMnO}_{4}(6 \mathrm{~g})$ as an oxidant, for $30 \mathrm{~min}$ at $30^{\circ} \mathrm{C}$. The graphene sheet was then washed with hot double distilled water (total $4 \mathrm{~L}$ ) by centrifugation and decantation until the $\mathrm{pH}$ was nearly neutral. The sample was filtered through a $0.2 \mu \mathrm{m}$ PTFE membrane. Finally it was washed with n-hexane and dried overnight under low pressure. The functionalized graphene was refluxed with $20 \mathrm{~mL}$ thionyl chloride for $24 \mathrm{~h}$ at $70^{\circ} \mathrm{C}$ to convert the surface bonded carboxylic acids to acylchloride. The $\mathrm{SOCl}_{2}$ was removed by distillation under low pressure and the resulting solid was dispersed in THF, and treated with BOC-L-alanine in the presences of pyridine for $12 \mathrm{~h}$ in an inert atmosphere. Finally, N-BOC group was deprotected by hydrolysis with trifloroacetic acid in dimethyl formamide. The resulting mixture was separated by centrifugation, washed with ethanol, and finally dried overnight under low pressure to yield L-alanine covalently immobilized on graphene sheet. FT-IR spectrum of functionalized graphene shows several peaks, viz, at $3405 \mathrm{~cm}^{-1}$ for $(\mathrm{O}-\mathrm{H})$, at $1725 \mathrm{~cm}^{-1}$ for $(\mathrm{C}=\mathrm{O})$, and peaks at $1360,1220,1050 \mathrm{~cm}^{-1}$, indicate respectively (C-O), epoxy, and alkoxy, respectively. While peaks at $1620 \mathrm{~cm}^{-1}$ and $1215 \mathrm{~cm}^{-1}$ confirm the presence of $(\mathrm{C}=\mathrm{C})$ and $(\mathrm{C}-\mathrm{OH})$, respectively. $(\mathrm{O})$, and $(\mathrm{OH})$ groups are not shown in the Scheme 1 , because the study was focused only on (-COOH) group, which was converted into -COCl by $\mathrm{SOCl}_{2}$. A new peak was encountered at $650 \mathrm{~cm}^{-1}$, which accounts for (C-Cl).

Notably, new peaks were observed at around 1248, 1310, 930, and $1640 \mathrm{~cm}^{-1}$, which reveal the presence of (-C-O-C-), (C-N), (C-C), and acid amide groups, respectively. This entire process was repeated for preparing all other catalysts. Hereafter, the catalysts are referred to as catalyst A, B, C and D where A, B, C and D are L-alanine, L-serine, L-valine, and L-arginine covalently immobilized on graphene sheet respectively.

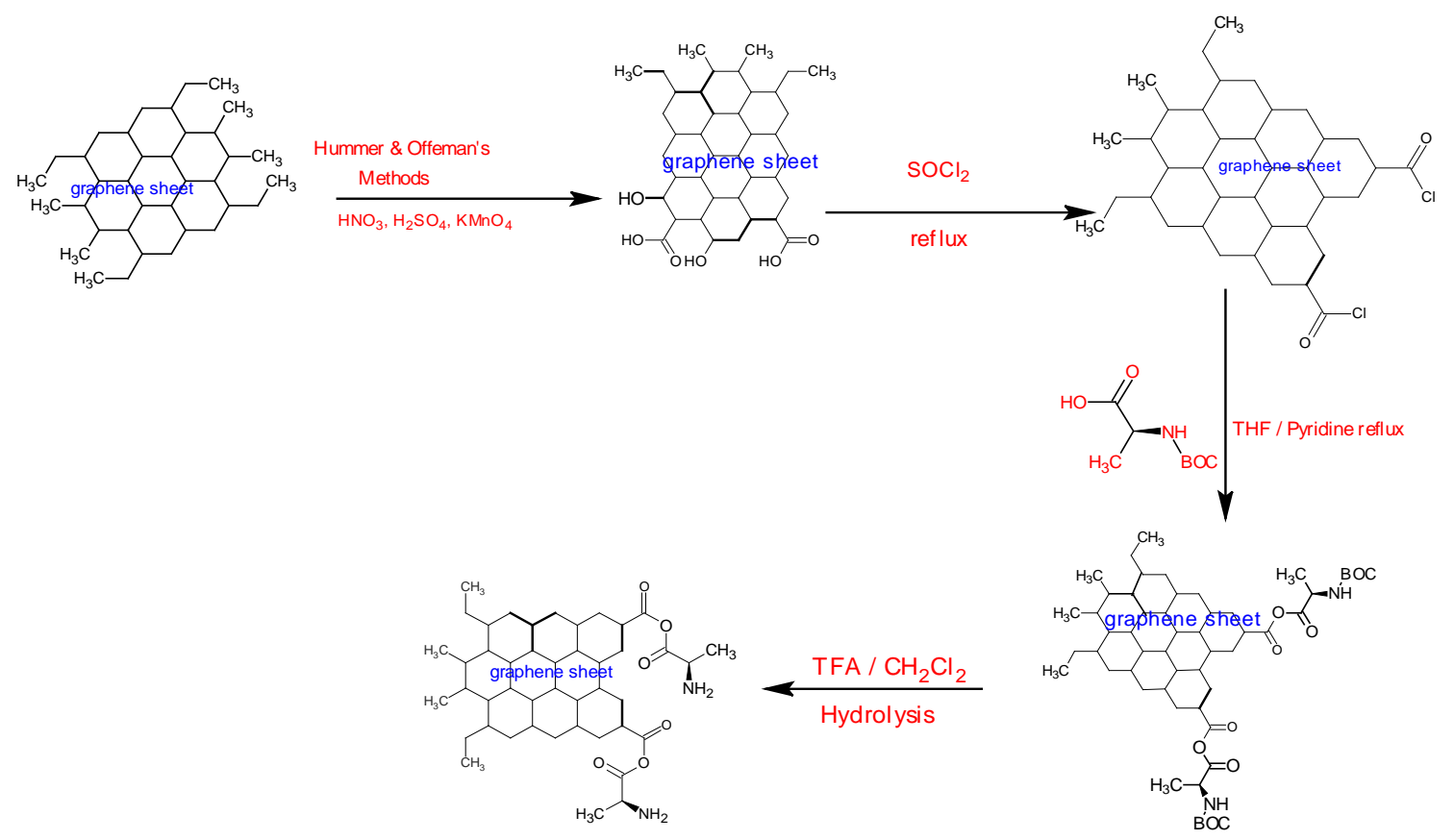

Scheme 1. A generalized scheme for covalently immobilizing amino acids on graphene sheet. 


\subsection{Catalytic Activity}

$50 \mathrm{mg}$ of catalyst, $10 \mathrm{~mL}$ distilled water, 3.3 equiv cyclohexanone and 1.0 equiv aldehyde were loaded to three necked batch reactor. The reaction mixture was stirred at $40^{\circ} \mathrm{C}$ for 24 hours as shown in Scheme 2. The reaction was periodically monitored by TLC $\left(\mathrm{R}_{\mathrm{f}}=0.27\right.$ for 2-(hydroxy(phenyl)methyl) cyclohexanone in ethyl acetate/n-hexane solvent system). Finally, the mixture was extracted with ethyl acetate. Column chromatography on silica gel using ethyl acetate/n-hexane (1:9) gave pure aldol product while anti/syn was determined by ${ }^{1} \mathrm{H}$ NMR analysis, and ee value was determined by Agilent 1100 HPLC with Daicel Chiralpak AS, OJ, and AD columns.

\section{Results and Discussion}

Remarkable improvement in the catalytic potential of linear amino acids has been attained by immobilizing them covalently on functionalized graphene. Therefore, a persistent struggle for developing new methodologies to synthesize chiral heterogeneous catalysts that could provide high \% yield and ee value, is underway [10]. We have been successful in developing a new pathway for the immobilization of linear amino acids covalently, on graphene sheet. The method comprises three-steps. The graphene sheet is first subjected to Hummer and Offeman's method of oxidation to develop (-COOH) and then these groups are converted into (-COCl) groups by treating with $\mathrm{SOCl}_{2}$. The second step involves the treatment of surface modified graphene with BOC-amino acid by refluxing with THF/pyridine. BOC-amino acid ensures that a covalent bond develops through the - $\mathrm{COOH}$ group of the amino acid and not through - $\mathrm{NH}_{2}$ group. In the last step, the amino acid is deprotected by hydrolysis with trifluoroacetic acid in DMF. In the covalent approach, the amino acids immobilized on graphene afford truly heterogeneous asymmetric catalysts. FTIR was used to elucidate the covalent immobilization between the hydroxyl group of BOC amino acids and the acyl-functionalized graphene sheet.

Catalysts A, B, C and D are capable of catalyzing the direct asymmetric aldol reactions in aqueous medium through enamine mechanism. The presence of water in the aldol reaction increases the rate of reaction in comparison to the reaction carried out under solvent free condition. An explanation to this effect is that the presence of water facilitates the hydrolytic detachment of amino acid from the resulting product of the aldol reaction [11]-[14]. The aldol reaction of cyclohexanone and benzaldehyde in the presence of catalyst "A" was chosen as a model reaction to evaluate the efficiency of catalyst A as a chiral heterogeneous catalyst. The result revealed that the desired aldol adduct was formed with a $85 \%$ yield, $94 \%$ ee and $6.7: 1 \mathrm{dr}$. The same reaction under the same conditions was also found to be catalyzed by catalysts "B" and " $C$ " and the results were (72\% yield, 93\% ee and 6:1 dr), and (84\% yield, 91\% ee and 16.5:1 dr), respectively. However, the same reaction under the same conditions was not very productive in the presence of the catalyst " $\mathrm{D}$ ", as the results were a low yield of $58 \%$, and an ee value of $10 \%$, indicating that the product is nearly a racemic mixture as presented in Figure 1.

These catalysts were also used for aldol reaction of cyclohexanone and m-nitrobenzaldehyde, affording both high \% yield and \% ee value as shown in Figure 2. The reaction under the same conditions in the presence of the catalyst "A" generated excellent results, viz, 94\% yield and 99\% ee value. These remarkable results appear probably due to the effect of nitro group attached to the benzaldehyde. These sumptuous results prompted to probe the efficiency of other catalysts for the same reaction. The results obtained with catalysts "B", "C" and "D" were ( $85 \%$ yield and $96 \%$ ee), ( $97 \%$ yield and $>99 \%$ ee) and ( $96 \%$ yield and $94 \%$ ee), respectively.

Experiments were extended further to study the catalytic efficiency of these catalysts for aldol reaction between cyclohexanone and 3-methylbutanal. Again the results were quite impressive both in terms of \% yield and ee value as shown in Figure 3. The catalyst "A" catalyzed the aldol reaction between cyclohexanone and

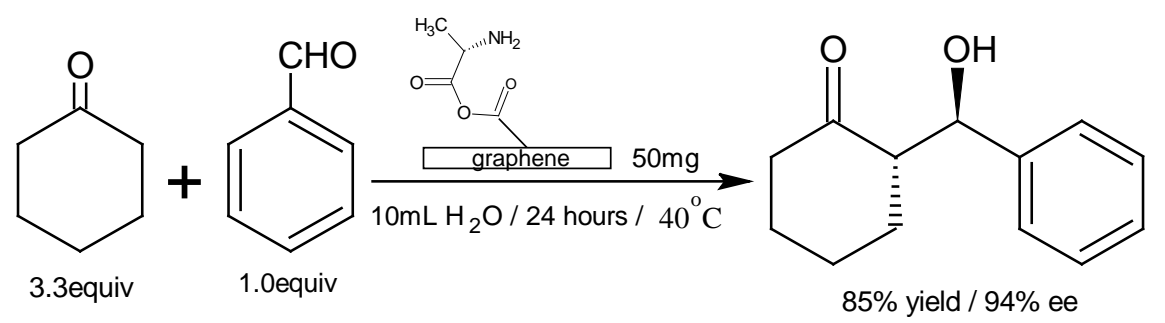

Scheme 2. Model reaction of cyclohexanone and benzaldehyde to 2-(hydroxy(phenyl) methyl) cyclohexanone, catalysed by L-alanine covalently immobilized on graphene. 


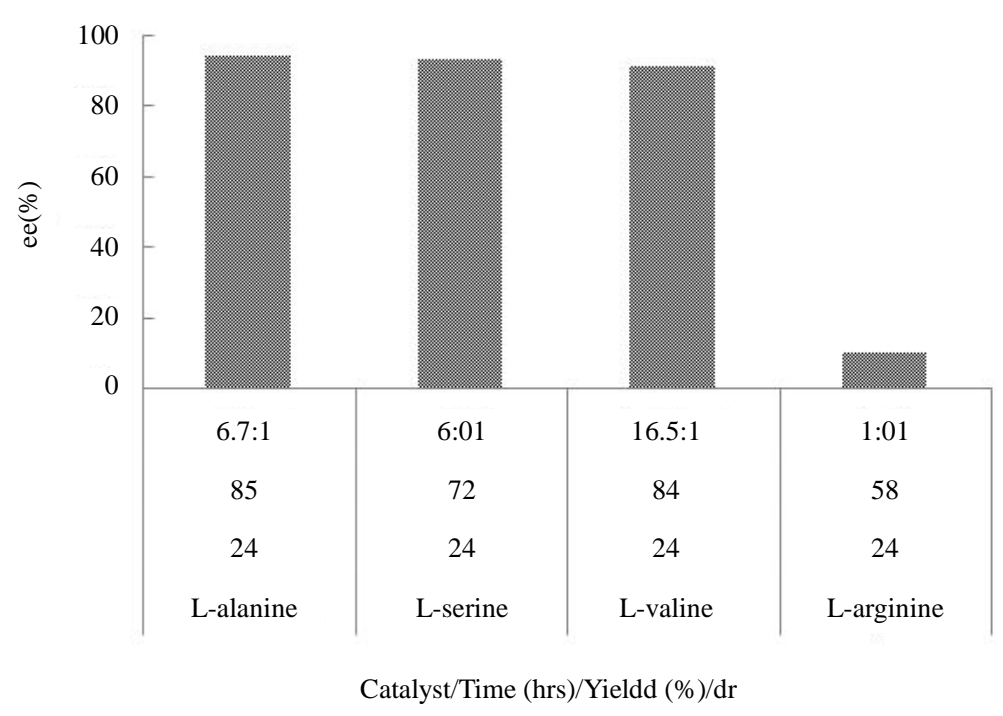

Figure 1. Catalytic efficiency of L-alanine, L-serine, L-valine, and L-arginine covalently supported on graphene sheet for asymmetric aldol reaction between cyclohexanone and benzaldehyde.

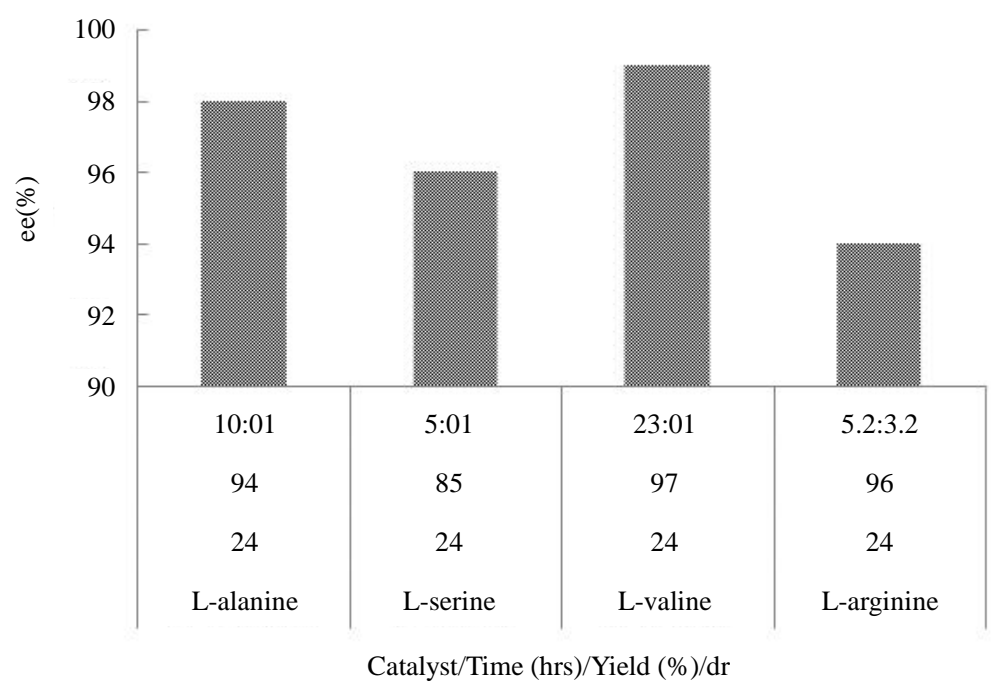

Figure 2. Effect of nitro group attached to benzaldehyde on \% yield and \% ee values in the aldol reaction of unmodified ketone with m-nitrobenzaldehyde, using L-alanine, L-serine, L-valine, and L-arginine covalently supported on graphene sheet as heterogeneous catalysts.

3-methylbutanal generating $65 \%$ yield and $80 \%$ ee while in the presence of catalysts "B", "C" and "D", the same reaction resulted in (53\% yield and 78\% ee), (58\% yield and $81 \%$ ee) and ( $44 \%$ yield and $56 \%$ ee).

The stereochemistry of the $\beta$-hydroxy group of the aldol adducts derived by L-linear amino acid supported on graphene sheet is R as demonstrated by chiral-phase HPLC analysis, optical rotation and compared with the literature [15]. The relative stereochemistry of cyclic aldol products was anti as determined by NMR spectroscopy and compared with literature [15].

Linear amino acid covalently immobilized on graphene sheet catalyzed asymmetric intermolecular aldol reaction of aldehyde and ketones through enamine route [16] as shown in the Scheme 3 . The donor ketone reacts with immobilized amino acid, resulting in enamine linkage. Next, the acceptor aldehyde combines with the chiral enamine. The resulting intermediate on hydrolysis gives enantiomerically enriched aldol product, and the catalyst is recycled. The presence of water promotes the rate of reaction probably by facilitating the hydrolysis 


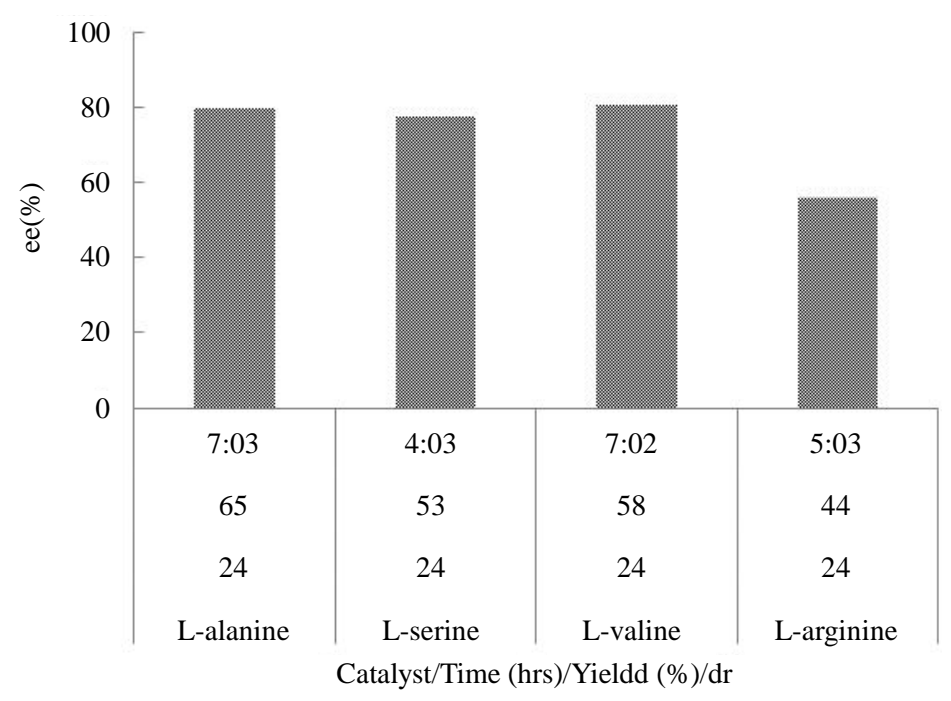

Figure 3. Efficiency of heterogenized L-alanine, L-serine, L-valine, and L-arginine on graphene sheet for aldol reaction between cyclohexanone and 3-methylbutanal.

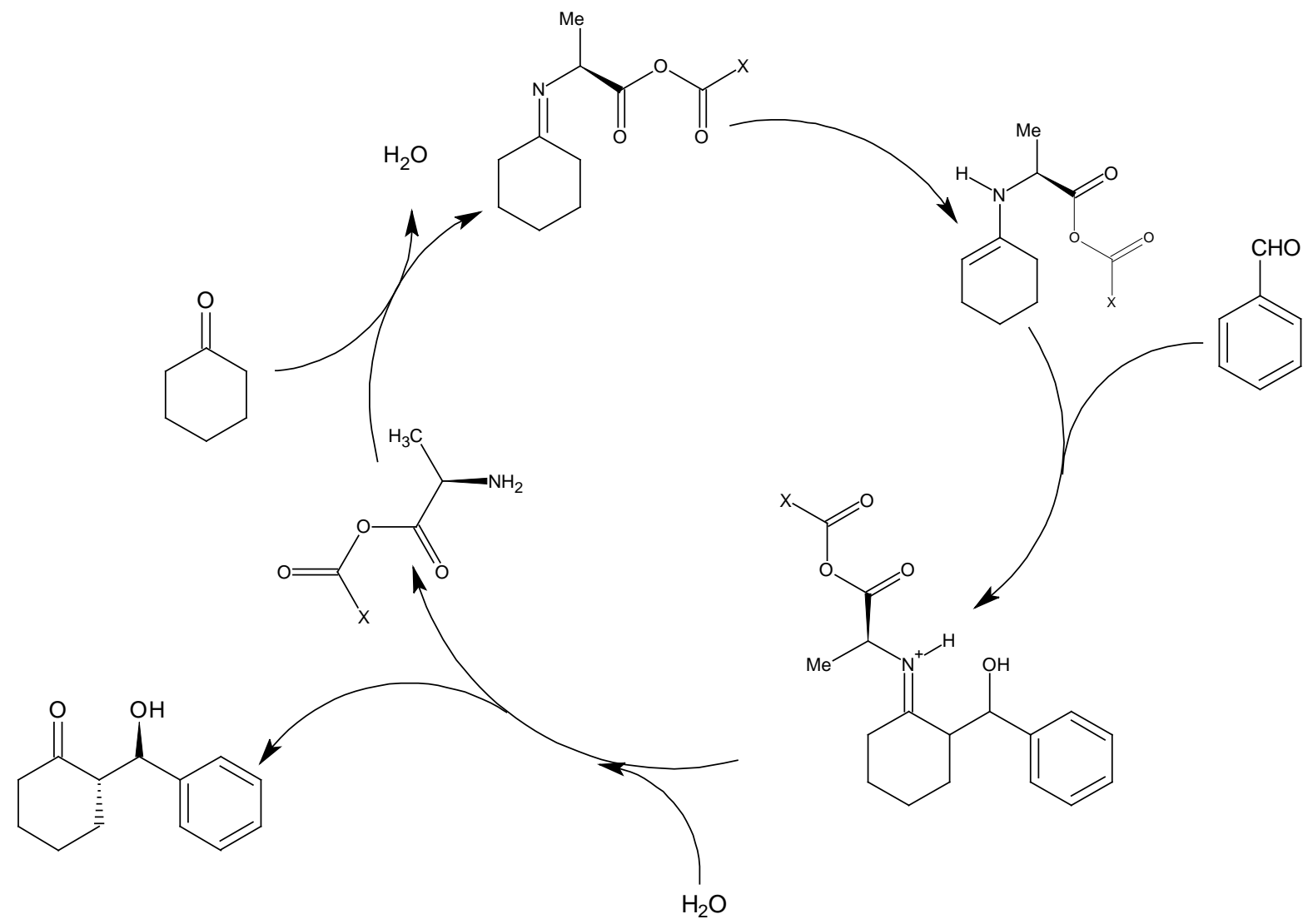

$\mathrm{X}$ : graphene sheet.

Scheme 3. Reaction route.

of the intermediate of the enamine catalytic cycle. These results provide clear evidence that acyclic amino acids covalently immobilized on graphene are remarkable chiral organo-catalysts, capable of catalyzing the direct asymmetric aldol reaction with excellent reactivity and stereoselectivity. Throughout the study, water was the only solvent. The catalysts showed good stability in solvent and recycled by simple filtration and used for five 
runs without coming across any loss in their catalytic efficiency.

\section{Conclusion}

Linear amino acids covalently supported on graphene sheet can function as remarkable heterogeneous organocatalysts for the direct asymmetric aldol reactions between cyclohexanone and aldehydes with excellent reactivity and stereoselectivity. All these catalysts generated high yield as well as high ee value for m-nitrobenzaldehyde in comparison with those for benzaldehyde and 3-methylbutanal. This improved efficiency of catalysts in the case of m-nitrobenzaldehyde most probably due to the presence of electron withdrawing group. In case of reaction between cyclohexanone and 3-methylbutanal, the catalysts manifest moderate \% yield and ee value. All these findings provide sufficient evidence for the effectiveness of linear amino acids supported on graphene sheet as useful, eco-friendly and heterogeneous catalysts for direct asymmetric aldol reactions.

\section{Acknowledgements}

The authors greatly acknowledge the financial support of High Education Commission of Pakistan, Project No. 20-1897/NRPU/R\&D/HEC/116806 and Project No: 20-1604/R\&D/092198, under National Research Programme for Universities.

\section{References}

[1] Amedjkouh, M. (2005) Primary Amine Catalyzed Direct Asymmetric Aldol Reaction Assisted by Water. Tetrahedron: Asymmetry, 16, 1411-1414. http://dx.doi.org/10.1016/j.tetasy.2005.02.031

[2] Yamada, Y., Yoshikawa, N., Sasai, H. and Shibasaki, M. (1997) Direct Catalytic Asymmetric Aldol Reactions of Aldehydes with Unmodified Ketones. Angewandte Chemie International Edition in English, 36, 1871-1873. http://dx.doi.org/10.1002/anie.199718711

[3] Dziedzic, P., Zou, W., Ibrahem, I., Sundén, H. and Córdova, A. (2006) Non-Linear Effects in Acyclic Amino AcidCatalyzed Direct Asymmetric Aldol Reactions. Tetrahedron Letters, 47, 6657-6661. http://dx.doi.org/10.1016/j.tetlet.2006.06.167

[4] List, B., Lerner, R.A. and Barbas, C.F. (2000) Proline-Catalyzed Direct Asymmetric Aldol Reactions. Journal of the American Chemical Society, 122, 2395-2396. http://dx.doi.org/10.1021/ja994280y

[5] An, Z., Zhang, W., Shi, H. and He, J. (2006) An Effective Heterogeneous L-Proline Catalyst for the Asymmetric Aldol Reaction Using Anionic Clays as Intercalated Support. Journal of Catalysis, 241, 319-327. http://dx.doi.org/10.1016/j.jcat.2006.04.035

[6] Yan, J. and Wang, L. (2009) Asymmetric Aldol Reactions Catalyzed by Efficient and Recyclable Silica-Supported Proline-Based Peptides. Chirality, 21, 413-420. http://dx.doi.org/10.1002/chir.20603

[7] Kassem, T., Jia, X., Zhu, X.X. and Lubell, W.D. (2010) Poly(vinyl alcohol)-Graft-Poly(ethylene glycol)-Supported Hydroxyproline Catalysis of Stereoselective Aldol Reactions. In: Macromolecular Symposia, Vol. 297, Wiley-VCH Verlag, 101-107. http://dx.doi.org/10.1002/masy.200900087

[8] Henseler, A.H., Ayats, C. and Pericàs, M.A. (2014) An Enantioselective Recyclable Polystyrene-Supported ThreonineDerived Organocatalyst for Aldol Reactions. Advanced Synthesis \& Catalysis, 356, 1795-1802. http://dx.doi.org/10.1002/adsc.201400033

[9] Marcano, D.C., Kosynkin, D.V., Berlin, J.M., Sinitskii, A., Sun, Z., Slesarev, A., Alemany, L.B., Lu, W. and Tour, J.M. (2010) Improved Synthesis of Graphene Oxide. ACS Nano, 4, 4806-4814. http://dx.doi.org/10.1021/nn1006368

[10] Itsuno, S. and Hassan, M.M. (2014) Polymer-Immobilized Chiral Catalysts. RSC Advances, 4, 52023-52043. http://dx.doi.org/10.1039/C4RA09561H

[11] Gruttadauria, M., Giacalone, F. and Noto, R. (2009) Water in Stereoselective Organocatalytic Reactions. Advanced Synthesis \& Catalysis, 351, 33-57. http://dx.doi.org/10.1002/adsc.200800731

[12] Paradowska, J., Stodulski, M. and Mlynarski, J. (2009) Catalysts Based on Amino Acids for Asymmetric Reactions in Water. Angewandte Chemie International Edition, 48, 4288-4297. http://dx.doi.org/10.1002/anie.200802038

[13] Maya, V., Raj, M. and Singh, V.K. (2007) Highly Enantioselective Organocatalytic Direct Aldol Reaction in an Aqueous Medium. Organic letters, 9, 2593-2595. http://dx.doi.org/10.1021/ol071013l

[14] Giacalone, F., Gruttadauria, M., Meo, P.L., Riela, S. and Noto, R. (2008) New Simple Hydrophobic Proline Derivatives as Highly Active and Stereoselective Catalysts for the Direct Asymmetric Aldol Reaction in Aqueous Medium. Advanced Synthesis \& Catalysis, 350, 2747-2760. http://dx.doi.org/10.1002/adsc.200800555 
[15] Córdova, A., Zou, W., Ibrahem, I., Reyes, E., Engqvist, M. and Liao, W.W. (2005) Acyclic Amino Acid-Catalyzed Direct Asymmetric Aldol Reactions: Alanine, the Simplest Stereoselective Organocatalyst. Chemical Communications, 2005, 3586-3588. http://dx.doi.org/10.1039/b507968n

[16] Pihko, P.M., Majander, I. and Erkkilä, A. (2009) Enamine Catalysis. In: Asymmetric Organocatalysis, Springer, Berlin Heidelberg, 145-200. http://dx.doi.org/10.1007/128_2008_21 